\title{
Probing the inner regions of MCG-06-30-15: a link between AGN activity and star formation?
}

\author{
S. I. Raimundo*1, R. I. Davies ${ }^{2}$, P. Gandhi ${ }^{3}$, A. C. Fabian ${ }^{4}$, R. E. A Canning ${ }^{4,5}$ and \\ V. D. Ivanov 6 \\ ${ }^{1}$ SISSA (International School for Advanced Studies), Italy \\ ${ }^{2}$ Max-Planck-Institut für extraterrestrische Physik, 85741 Garching, Germany \\ ${ }^{3}$ Institute of Space and Astronautical Science (ISAS), JAXA, 3-1-1 Yoshinodai, chuo-ku, \\ Sagamihara, Kanagawa 229-8510, Japan \\ ${ }^{4}$ Institute of Astronomy, Madingley Road, Cambridge CB3 OHA \\ ${ }^{5}$ Kavli Institute for Particle Astrophysics and Cosmology, Stanford University, 452 Lomita Mall \\ Stanford, CA 94305-4085 USA \\ ${ }^{6}$ European Southern Observatory, Ave. Alonso de Cordova 3107, Vitacura, Santiago 19001, \\ Chile \\ E-mail: sandra.raimundodsissa.it
}

In this contribution we present the first observations using integral field spectroscopy of the galaxy MCG-6-30-15, a Narrow Line Seyfert 1 galaxy with high black hole accretion rate and well studied X-ray properties. This galaxy is therefore a suitable test case to study the relation between black hole accretion and the properties of the inner regions of the host galaxy.

We are able to remove the dominant spectral contribution of the broad brackett emission lines and trace the distribution and kinematics of the stars and also the [Fe II] line emission. We find that there is a counter-rotating stellar core with a radius $\mathrm{r}<125 \mathrm{pc}$, which appears to be associated with the [Fe II] emission. Based on the presence of this emission line, we estimate the age of the central stellar population to be of order of $65 \mathrm{Myr}$. We show that the gas needed to fuel the black hole is, at most, only 1 per cent of that needed to form these stars.

Nuclei of Seyfert galaxies and QSOs - Central engine \& conditions of star formation November 6-8, 2012

Max-Planck-Insitut für Radioastronomie (MPIfR), Bonn, Germany

\footnotetext{
*Speaker.
} 


\section{Introduction}

MCG-6-30-15 is an elongated lenticular (S0) galaxy and classified as a Seyfert 1.2. This galaxy is best-known for having provided the first detection of a relativistic broadened $\mathrm{Fe} \mathrm{K}_{\alpha}$ emission line generated in the inner regions of an accretion disc [22], hence providing evidence for the presence of a supermassive black hole. MCG-6-30-15 has been studied in several wavelengths. The Active Galactic Nucleus (AGN) has been studied extensively in the X-rays, it presents a luminosity of $\mathrm{L}_{\mathrm{X}}(2-10 \mathrm{keV}) 4 \times 10^{42} \mathrm{erg} \mathrm{s}^{-1}$ and there is evidence of a warm absorber [18]. The nonstellar continuum can also be observed in the infrared [14] and it is detected but only marginally resolved in the radio [13]. Despite the studies mentioned above, the mass of the black hole responsible for the AGN activity is still not well constrained. The current estimates using stellar velocity dispersion measurements, X-ray variability and the luminosity of the galaxy have found the mass to be in the range: $(0.3-3) \times 10^{7} \mathrm{M}_{\odot}[10,1,23]$. The stellar population is dominated by an old population $(\sim 10 \mathrm{Gyr})$ but with indication of a series of previous bursts of star formation distributed in age among the younger stellar population $(<500 \mathrm{Myr})[3]$.

The advent of integral field spectroscopy (IFS) has opened a new perspective on the black hole fuelling process and on the relation between the stellar properties and the AGN activity. MCG-630-15 is one of the key galaxies to understand the accretion physics, useful in particular to study black hole fuelling at higher accretion rates. We observed the inner regions of this galaxy, with the main goal of studying its stellar and dynamical properties. The results presented here provide a new perspective on MCG-6-30-15, and on the properties of its central black hole.

\section{Stellar kinematics and gas dynamics}

Using the near-IR integral field spectrograph SINFONI we obtain a map of the central 3" $\mathrm{x}$ 3" of MCG-6-30-15. For this galaxy, the broad hydrogen Brackett AGN emission lines are very strong in the central pixels, making the stellar absorption features hard to identify. As a first step, we fit the broad emission lines at each pixel by fixing their relative intensities and wavelengths and subtract them from the observed spectra.

The velocity map and stellar velocity dispersion distribution are determined from our fully reduced and broad line subtracted data cube, by fitting the spectra with the IDL routine pPXF [5]. We use the strength of the absorption lines and the 2D Voronoi binning routine [4], to bin the data spatially to a minimum signal-to-noise $\mathrm{S} / \mathrm{N}=5$. An example of our spectra (in this case the integrated spectrum) is shown in Fig. 1. Each spaxel is then fitted in the wavelength range $\lambda_{\text {rest }}(1.57-1.716) \mu \mathrm{m}$, to obtain a map of stellar velocity and velocity dispersion. The results show a low rotational velocity when compared with the velocity dispersion (Fig. 2). Negative velocities are associated with blue-shifted absorption lines and positive velocities with redshifted absorption lines. The inner and outer regions of our map indicate two different and counter-rotating components, with the $r<0.8^{\prime \prime} \sim 125 \mathrm{pc}$ region rotating clockwise and the $r>0.8^{\prime \prime}$ rotating counterclockwise. The rotation of the counter rotating core is clearly seen in the northern (yellow/red zone) and eastern (blue zone) regions of the velocity map. The velocity dispersion map presents values that decrease inwards and are constant at the centre. 


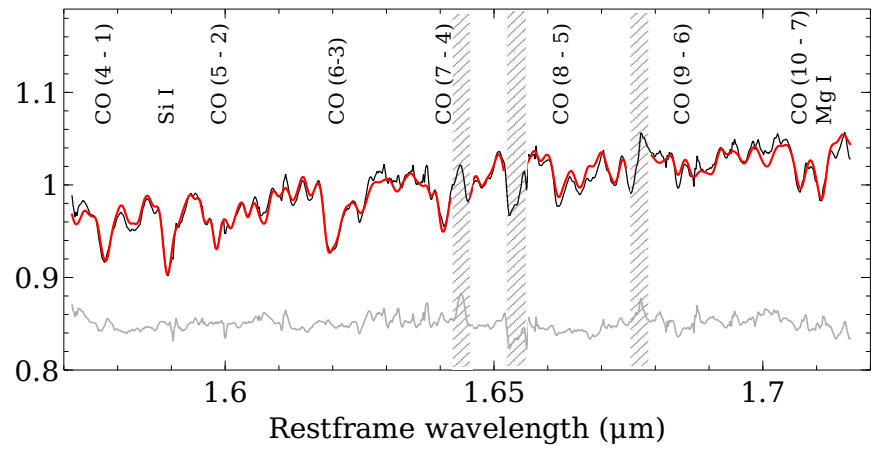

Figure 1: Spectrum and fitting results versus rest-frame wavelength. In black, integrated spectrum over a radius of $\sim 1.1^{\prime \prime}$ excluding the central $6 \times 4$ pixels. In red, pPXF best fit combination of stellar templates and 4th order polynomial (to model the AGN continuum). The residuals are shown in grey and have been offset from zero for plotting purposes. The vertical shaded regions were excluded from the fit.

The general kinematic properties mentioned above, indicate that we are most likely in the presence of a kinematically distinct core. These type of systems are fairly common in ellipticals but counter-rotating systems are rarer $(<10$ per cent) in S0 type galaxies $[9,8]$. The drop in the velocity dispersion occurs for $r<0.8^{\prime \prime}$ and is possibly related with the counter-rotating core. This phenomenon has been observed for another S0 galaxy with a kinematically-distinct core, NGC 7332, where the velocity dispersion increases from $r \sim 10^{\prime \prime}$ or $1 \mathrm{kpc}$ towards the centre of the galaxy, but drops by $\sim 10 \mathrm{~km} / \mathrm{s}$ when in the region of the counter-rotating core [7]. Our data cover a smaller radii $\left(r_{\max } \sim 1.4^{\prime \prime}\right.$ or $200 \mathrm{pc}$ ) than the work of Falcon-Barroso et al. [7], which does not permit us to evaluate if the velocity dispersion decreases again as we move to larger radii. The dynamically decoupled core could be the result of inflow of gas into the central regions of the galaxy via, for example, a past minor merger event. For our target, the majority of the stellar population is old and fairly homogeneous, not showing any variations with radius [2]. There is nevertheless indication of a series of previous star formation bursts [3]. The evolution for this galaxy is not expected to be through major mergers, since only about $2-2.5$ per cent of S0 galaxies are expected to have had a major merger in their past [16]. On the other hand, secular evolution is expected to dominate the evolution of Narrow Line Seyfert 1 galaxies such as MCG-6-30-15 (e.g. [15]).

The gas is traced by the $\lambda=1.644 \mu \mathrm{m}$ forbidden emission line of [Fe II], the strongest emission line in the spectra. We show the two-dimensional line properties in the three panels of Fig. 3. In these plots, the velocities are measured in relation to the systemic velocity of the galaxy. The lines show an asymmetry in the intensity and a velocity gradient along the major axis of the galaxy. Since the signal in the data is weak, we cannot say with certainty what causes this [Fe II] emission, but we argue that it is caused by supernova shocks and not AGN outflows.

The velocity map for [Fe II] shows a deviation from the rotation velocity of the stars and an elongation along the major axis of the galaxy, as seen in [O III] $5007 \AA$ emission using HST observations ([20]). However, the [Fe II] velocity offset from the rotational velocity is not very large, and could be due to uncertainties in the velocity measurements. Also, the transition in velocity along the major axis is fairly smooth and resembles a rotation pattern. The asymmetry in 

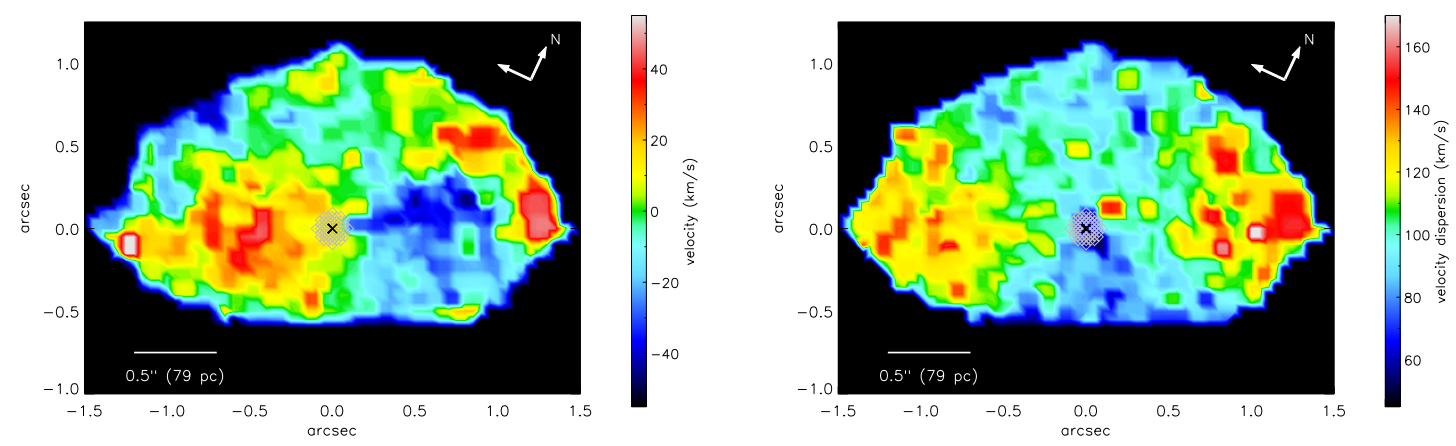

Figure 2: Two-Dimensional map of line of sight velocity and the velocity dispersion from the spectral fitting with pPXF. Top: Line of sight velocity map. To note that the values are more uncertain in the central $\sim 4 \times 4$ pixels due to the presence of a high AGN continuum. Bottom: Map of velocity dispersion. Individual pixels in the regions where the flux decreases to $1 / 125$ of its peak value were masked out and excluded from the analysis, which correspond to the outer regions of our field-of-view. The errors are measured using a Monte Carlo approach: $\pm 4.5 \mathrm{~km} / \mathrm{s}$ for the mean line-of-sight velocity and $\pm 5.1 \mathrm{~km} / \mathrm{s}$ for the velocity dispersion.

[O III] emission is on a larger scale than the field-of-view of our observations, and we cannot check if the $[\mathrm{Fe} \mathrm{II}]$ and $[\mathrm{O} \mathrm{III}]$ emission are tracing the same outflow geometry.

The $[\mathrm{Fe} \mathrm{II}]$ emission is excited by electron collisions and is a good tracer of shocks. In the case of MCG-6-30-15, the extended emission observed could be caused by nuclear mass outflow shocks with ambient clouds, or by supernova-driven shocks [12]. From the arguments above, we propose that the [Fe II] emission is most likely due to supernova remnants shock fronts and therefore can be used as a tracer of the supernova rate $([11,19])$. In this case, the [Fe II] emission can be used to investigate the properties of the stellar population of MCG-6-30-15.

\section{Black hole and central stellar population}

It is possible that the counter-rotating core is associated with new inflow of gas which fuelled the formation of a new stellar population with distinct stellar kinematics. The [Fe II] emission traces the supernova and is observed in the inner regions where the counter-rotating core is. We used the measured [Fe II] flux and the STARS code [21, 6] to learn more about the episode of star formation and its relation with the black hole fuelling. The starburst has an age of approximately $6.5 \times 10^{7} \mathrm{yr}$, formed $2 \times 10^{8} \mathrm{M}_{\odot}$ stars and presents a mass loss rate of $1 \mathrm{M}_{\odot} \mathrm{yr}^{-1}$. If the AGN activity is fuelled by ejections from the stars, we would need a mass loss comparable to the mass accretion rate of the black hole, which is $0.022 \mathrm{M}_{\odot} \mathrm{yr}^{-1}$. The mass loss rate obtained using STARS would be enough to fuel the black hole activity, providing that the process is efficient. On the other hand, if the new inflow of gas was responsible for the star formation and for providing the fuel to the black hole, we would need an amount of gas capable of powering the AGN for $6.5 \times 10^{7} \mathrm{yr}$. Considering that the black hole was always active and at a constant mass accretion rate, the total initial gas mass needed for black hole fuelling would be $1.4 \times 10^{6} \mathrm{M}_{\odot}$ which is roughly 1 per cent of the total mass used to form stars. These calculations provide approximate values only, but are useful to understand the general mass budget in the central region of the galaxy. In either case, it 

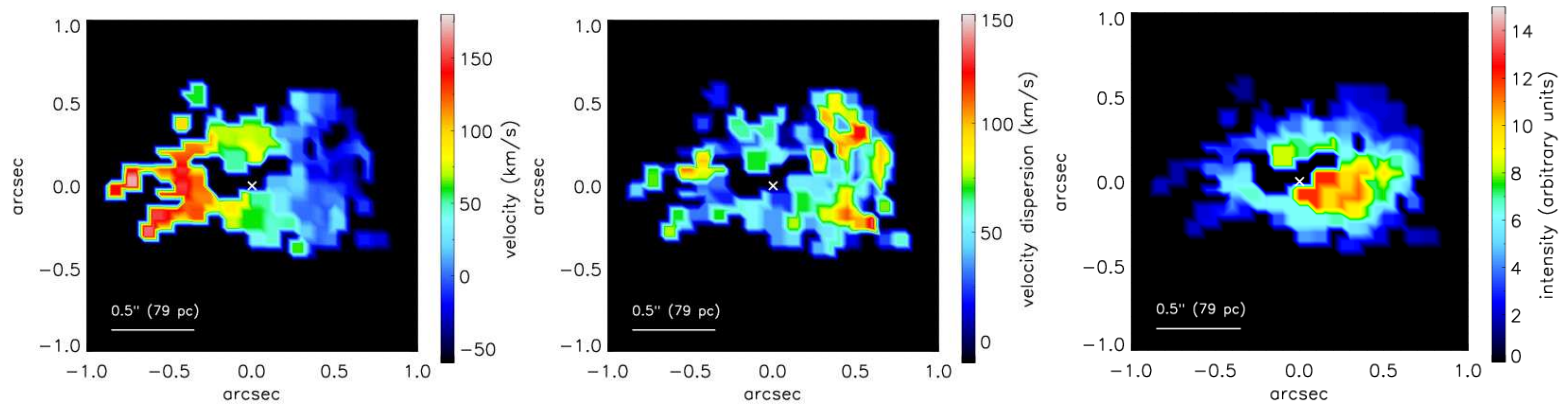

Figure 3: Panels showing the [Fe II] emission properties of MCG-6-30-15 obtained by fitting the line with MPFIT. The white cross marks the position of the AGN. Bins with $\mathrm{S} / \mathrm{N}<3$ and bins for which the velocity dispersion is lower than the instrumental broadening were excluded (black regions). Top panel: Velocity in $\mathrm{km} / \mathrm{s}$. Middle panel: Velocity dispersion in $\mathrm{km} / \mathrm{s}$. Bottom panel: Line intensity.

is, nevertheless, still uncertain how this mass can move inwards to $<1 \mathrm{pc}$ scales to fuel the black hole.

To obtain a quantitative limit on the black hole mass, we measure the average velocity dispersion and line-of-sight velocity in the inner $r<0.2^{\prime \prime}$ region, which gives an average of $\sigma \sim 89$ $\mathrm{km} / \mathrm{s}$. The enclosed mass is then an upper limit for the black hole mass, and we have $M_{\mathrm{BH}}<$ $\left(v^{2}+3 \times \sigma^{2}\right) R / G$. We can also estimate the enclosed mass due to young stars, by integrating the luminosity in the $r<0.2^{\prime \prime}$ region and using the M/L ratio from STARS. Subtracting the enclosed stellar mass from the dynamical mass we get an upper limit of $M_{\mathrm{BH}}<1.5 \times 10^{8} \mathrm{M}_{\odot}$.

\section{Conclusions}

This contribution focused on the properties of the inner $\sim 470 \mathrm{pc}$ of the galaxy MCG-6-30-15 and on the relation between the central stellar population and the accretion history of the black hole. We argue that there is a counter-rotating stellar core extending out to $125 \mathrm{pc}$, associated with the [Fe II] emission. From these observations we were able to calculate the properties of the central stellar population, including its age and stellar mass. Assuming that the counter-rotating core is a result of a recent inflow of gas, we determine that the percentage of gas used to fuel the black hole is at most 1 per cent of the gas used to form stars. From the dynamical properties measured at $\mathrm{r}<0.2^{\prime \prime}$ and the mass-to-light ratio we calculate an upper limit for the black hole mass of $M_{\mathrm{BH}}<1.5 \times 10^{8} \mathrm{M}_{\odot}$.

The content of this contribution is presented in more detail in Raimundo et al. [17].

\section{References}

[1] N. Bennert, B. Jungwiert, S. Komossa et al. Size and properties of the narrow-line region in Seyfert-1 galaxies from spatially-resolved optical spectroscopy. A\&A, 2006. 459:55-69.

[2] C. Boisson, S. Coupé, J. G. Cuby et al. Near IR spectroscopy of active galactic nuclei. A\&A, 2002. 396:489-501. 
[3] C. Bonatto, E. Bica, M. G. Pastoriza et al. Star formation in distant starburst galaxies. A\&A, 2000. 355:99-112.

[4] M. Cappellari and Y. Copin. Adaptive spatial binning of integral-field spectroscopic data using Voronoi tessellations. MNRAS, 2003. 342:345-354.

[5] M. Cappellari and E. Emsellem. Parametric Recovery of Line-of-Sight Velocity Distributions from Absorption-Line Spectra of Galaxies via Penalized Likelihood. PASP, 2004. 116:138147.

[6] R. I. Davies, F. Müller Sánchez, R. Genzel et al. A Close Look at Star Formation around Active Galactic Nuclei. ApJ, 2007. 671:1388-1412.

[7] J. Falcón-Barroso, R. F. Peletier, E. Emsellem et al. Formation and evolution of SO galaxies: a SAURON case study of NGC 7332. MNRAS, 2004. 350:35-46.

[8] D. Krajnović, E. Emsellem, M. Cappellari et al. The ATLAS ${ }^{3 D}$ project - II. Morphologies, kinemetric features and alignment between photometric and kinematic axes of early-type galaxies. MNRAS, 2011. 414:2923-2949.

[9] K. Kuijken, D. Fisher and M. R. Merrifield. A search for counter-rotating stars in S0 galaxies. MNRAS, 1996. 283:543-550.

[10] I. M. McHardy, K. F. Gunn, P. Uttley et al. MCG-6-30-15: long time-scale X-ray variability, black hole mass and active galactic nuclei high states. MNRAS, 2005. 359:1469-1480.

[11] A. F. M. Moorwood and E. Oliva. Infrared spectroscopy of forbidden Fe II, H2, and H line emission in galactic nuclei. A\&A, 1988. 203:278-288.

[12] H. Mouri, K. Kawara and Y. Taniguchi. Excitation Mechanism of Near-Infrared [Fe II] Emission in Seyfert and Starburst Galaxies. ApJ, 2000. 528:186-200.

[13] C. G. Mundell, P. Ferruit, N. Nagar et al. Radio Variability in Seyfert Nuclei. ApJ, 2009. 703:802-815.

[14] E. Oliva, L. Origlia, R. Maiolino et al. Starbursts in active galaxy nuclei: observational constraints from IR stellar absorption lines. A\&A, 1999. 350:9-16.

[15] G. Orban de Xivry, R. Davies, M. Schartmann et al. The role of secular evolution in the black hole growth of narrow-line Seyfert 1 galaxies. MNRAS, 2011. 417:2721-2736.

[16] O. H. Parry, V. R. Eke and C. S. Frenk. Galaxy morphology in the $\Lambda$ CDM cosmology. MNRAS, 2009. 396:1972-1984.

[17] S. I. Raimundo, R. I. Davies, P. Gandhi et al. The Black Hole and Central Stellar Population of MCG-6-30-15. Accepted for publication in MNRAS, 2013.

[18] C. S. Reynolds, A. C. Fabian, K. Nandra et al. ASCA PV observations of the Seyfert 1 galaxy MCG-6-30-15: rapid variability of the warm absorber. MNRAS, 1995. 277:901-912. 
[19] M. J. F. Rosenberg, P. P. van der Werf and F. P. Israel. [FeII] as a tracer of supernova rate in nearby starburst galaxies. A\&A, 2012. 540:A116.

[20] H. R. Schmitt, J. L. Donley, R. R. J. Antonucci et al. A Hubble Space Telescope Survey of Extended [O III] $\lambda 5007$ Emission in a Far-Infrared Selected Sample of Seyfert Galaxies: Observations. ApJS, 2003. 148:327-352.

[21] A. Sternberg. The Initial Mass Functions in the Super-Star Clusters NGC 1569A and NGC 1705-1. ApJ, 1998. 506:721-726.

[22] Y. Tanaka, K. Nandra, A. C. Fabian et al. Gravitationally redshifted emission implying an accretion disk and massive black hole in the active galaxy MCG-6-30-15. Nat, 1995. 375:659661.

[23] R. V. Vasudevan, R. F. Mushotzky, L. M. Winter et al. Optical-to-X-ray emission in lowabsorption AGN: results from the Swift-BAT 9-month catalogue. MNRAS, 2009. 399:15531575 . 